\title{
HIV/STIs and Pregnancy Prevention, Using a Cervical Barrier and Microbicide
}

\author{
Alfred A. Shihata ${ }^{1}$, Steven A. Brody ${ }^{2}$ \\ ${ }^{1}$ Scripps Institution of Medicine and Science, San Diego, USA; ${ }^{2}$ Department Endocrinology \& Metabolism, School of Medicine, \\ University of California, La Jolla, USA. \\ E-mail: alfred@femcap.com,femcap@yahoo.com
}

Received August 5 ${ }^{\text {th }}$, 2011; revised September 27 ${ }^{\text {th }}$, 2011; accepted October $13^{\text {th }}, 2011$.

\begin{abstract}
Objectives: 1) HIV prevention, using a mechanical cervical barrier in combination with microbicide. 2) Prevention of pregnancy. 3) Shield the cervix to prevent sperm penetration and Gonorrhea, Chlamydia and HIV virus invasion. Methods: We investigated a new FDA approved cervical barrier FemCap (Figure 1). The FemCap is a contraceptive device that is designed with a unique delivery system for microbicides on its cervical and vaginal sides (Figure 2) to ensure better coverage, and retention of gel on the cervix and vagina. We also compared the acceptability and adherence with the FemCap, and retention of a stained vaginal lubricant when delivered with the FemCap versus the vaginal lubricant when delivered using a traditional vaginal applicator (Figure 3). We used the same vaginal applicator utilized in the CAPRISA 004 [1] study, to deliver Tenofovir microbicide. Thirty women compared the use of a vaginal applicator to deliver a high viscosity stained vaginal lubricant before and after intercourse, versus the FemCap to deliver the same lubricant once before intercourse. The acceptability and efficacy of this delivery system was evaluated. Results: Forty percent (12) women missed the application of the lubricant with the vaginal applicator before intercourse and $10 \%$ missed it after intercourse. Amongst FemCap users (3) women (10\%) missed application of the vaginal lubricant before intercourse and all of them inserted it after intercourse. The stained gel was better retained over the cervix (Figure 4) by single application with the FemCap versus two applications with the traditional applicator (Figure 3). Conclusions: Women in this study preferred the FemCap due to elimination of leakage and the single application, method versus two applications with the traditional vaginal applicator. The use of the FemCap, can prevent pregnancy, HIV mother-to-child transmission, enhance compliance and retention of gel over the cervix and vagina that may potentially prevent STIs and increase the efficacy of Tenofovir.
\end{abstract}

Keywords: HIV Prevention, Microbicide Tenofovir, FemCap Delivery System

\section{Introduction}

Despite numerous effective evidence-based preventive interventions, such as the condom [2,3], circumcision, Tenfovir gel [1], the HIV pandemic is still outpacing all the above methods. Therefore the importance of innovative practices employing the above mentioned methods as well as novel interventions are needed.

Women are the fastest growing population affected by sexually transmitted infections and Acquired Immune Deficiency Syndrome (STIs/AIDS) worldwide [4]. Women are biologically vulnerable, and suffer disproportionately more from the consequences of unplanned pregnancy and STIs/HIV infections.

The cervix is the main portal of entry for the HIV virus. This is due to the presence of high concentration of the
Chemokine receptors CCR5 and CRX4 on the cervix [5]. Gonorrhea and Chlamydia are intracellular organisms and they invade and colonize the endocervical canal. These non-ulcerative STIs, when present, enhance the invasion of HIV [6]. Covering the cervix by a cervical barrier in combination of spermicide/microbicide can 1) prevent sperm penetration 2) potentially prevent Gonorrhea Chlamydia, and 3) potentially prevent HIV virus invasion of the cervix.

With no cure or vaccine anticipated in the near future, HIV continues to spread despite the availability of the condom and other preventive measures. Vaginal microbicides are a female-initiated new class of drugs that are intended to prevent the transmission of STIs/HIV from the male to the female and from the female to the male. They come in gels, creams, suppositories, films, and tablets. 
Almost all microbicides, even lemon juice and soap and water, can kill the fragile HIV virus in-vitro. Some microbicides when inserted into the vagina, including, Nonoxynol-9 [7], Cellulose Sulfate [8], Pro 2000 [9] and Carragaurd [10] failed to prevent HIV/AIDS infections in clinical trials.

Tenofovir gel, a nucleotide reverse transcriptase inhibitor, was shown to significantly reduce the risk for HIV transmission in CAPRISA 004 [1]. Tenofovir vaginal gel reduced the likelihood of HIV acquisition by an estimated $39 \%$ overall. Among women with good (>80\%) (selfreported), compliance, HIV seroconversion was 54\% lower in the Tenofovir arm compared with the placebo $\operatorname{arm}(\mathrm{P}=0.025)$. Among those who had poor adherence (self-reported) with Tenofovir application $(<50 \%)$, the reduction in HIV seroconversion in the Tenofovir arm was only $28 \%$. Tenofovir $1 \%$ gel would have far less systemic absorption, side effects or developing resistance and would have much higher concentration in the vagina if compared with oral antiretroviral pre-exposure prophylaxis. To improve on our previous study [11] and to enhance detection of the vaginal lubricant we, mixed two drops of Gentian Violet dye with every $10 \mathrm{ml}$ of the lubricant, and also increased the number of the participants.

This research in progress to validate and verify the result of our previous published article [11] .It should be noted that the addition of the Gentian violet dye was a major improvement over our previous research. The addition of the dye greatly enhanced the detection of retained gel over the cervix and vagina (Figure 4). This clearly distinguished the absence of the gel when applied with the traditional vaginal applicator (Figure 5). We considered the increase of participants from 20 to 30 is also a major improvement, which markedly increased the power of the study. This is despite the difficulty of recruitment of women to use a stained gel.

\section{Methods}

This is work in progress to validate our previous pilot study [11] to compare the adherence, retention and efficacy of two microbicide delivery systems. The FemCap and the conventional vaginal applicator used in the CAPRISA 004 study to deliver the tenofovir gel.

The FemCap is a new contraceptive device, (Figure 1) [12-19] that is designed with a unique delivery system for the microbicide on its cervical and vaginal sides (Figure 2). The aim of this design is to allow for better adherence, coverage, distribution and retention of the gel for up to 24 hours.

We intended to build upon the partial success of the Tenofovir gel $1 \%$ in prevention of HIV acquisition. We investigated the feasibility and acceptability of the FemCap microbicide delivery system as an alternative to the traditional vaginal applicator that was used in CAPRISA 004 [1] ((Figure 3). Participants selected for this study were ages 18 to 40 years, were not pregnant, and did not desire to be pregnant during the 4 week study duration. All subjects were free from sexually transmitted diseases and reported being sexually active. Thirty participants (40\% of whom were sterilized and $60 \%$ of whom were on hormonal contraception), signed an informed consent and underwent a complete physical and pelvic exam as well as cervical colposcopic evaluation.

Fifteen women (Group A) were randomly assigned to use the vaginal applicator, (Figure 3) and the other 15 women (Group B) were assigned to use the FemCap (Figure 2) to deliver a high viscosity vaginal lubricant stained with Gentian violet dye, as a substitute for Tenofovir gel for two weeks.

After one week of wash over group (A) then crossed over and used the FemCap and group (B) used the vaginal applicator for two additional weeks. Participants were instructed to record and report the use of the vaginal applicator and the FemCap and any side effects on a calen-

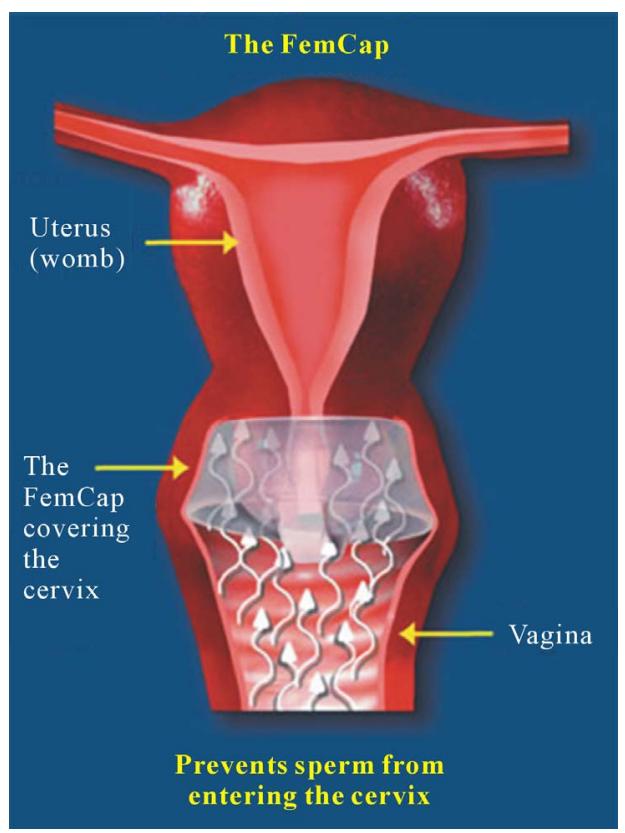

Figure 1. New FDA approved cervical barrier FemCap.

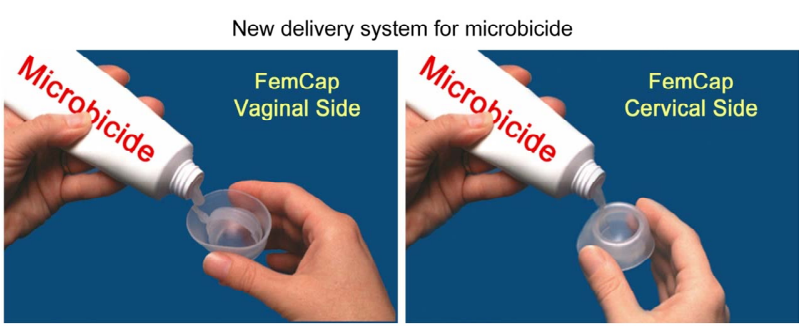

Figure 2. FemCap cervical and vaginal sides. 
dar. This facilitated a comparison between compliance with the vaginal applicator to deliver a vaginal lubricant before and after intercourse and with the FemCap to deliver the same lubricant only once before intercourse for four weeks. Women in both groups had an average three acts of intercourse per week.

We provided participants with hygienic pads to monitor the expulsion of the stained gel.

We swabbed each participant's vagina and introitus for the presence or absence of the stained lubricant at 6 , and 12 , hours after insertion of the lubricant using both devices. In addition cervical colposcopic exam was performed and the cervix was swabbed 24 hours after insertion of the lubricant using the applicator and the FemCap. Finally the investigators conducted in-depth interviews with each of these 30 women as to what they thought the reasons might be why they inserted or failed to insert gel.

\section{Results}

None of the participants reported any significant side effects using either device. Naked eye examination and colposcopy did not show any significant lesions over the cervix or the vagina. Women reported increased leakage of the stained lubricant from the vagina following the use of the vaginal applicator. This was verified by the presence of the stained gel on the hygienic pads.

Participants preferred the use of the FemCap, due to elimination of leakage and the single application protocol, versus two applications with the vaginal applicator. Women also stated that it was much easier to remember one application with the FemCap for a span of 24 hours, versus two applications, one before intercourse, (which was unpredictable) and a second application after intercourse.

Twelve women (40\%) reported that they missed the application of the stained lubricant more than once before intercourse by using the vaginal applicator and three different women (10\%) missed it after intercourse. Nine women $30 \%$ failed to apply the lubricant with the vaginal applicator after the cross over. With the FemCap, only three women (10\%) missed application of the stained vaginal lubricant before intercourse and two different women missed it after the cross-over Swabbing and colposcopy of the cervix and vagina after 6,12 and 24 hours, in women who used the FemCap and the vaginal applicator showed the following: The stained vaginal lubricant was present over the cervix (Figure 4), and to a lesser degree on the upper vagina with many bare areas, and was absent over the lower vagina and introitus 24 hours after application with the FemCap. We failed however to detect the presence of the stained vaginal lubricant after 12 hours on the cervix or the vagina (Figure 5) in women who used the vaginal applicator Table $\mathbf{1 .}$
Therefore according to this study, a single application of the stained vaginal lubricant using the FemCap delivery system has proven to have better acceptability, compliance, gel distribution, and retention than two applications by the vaginal applicator.

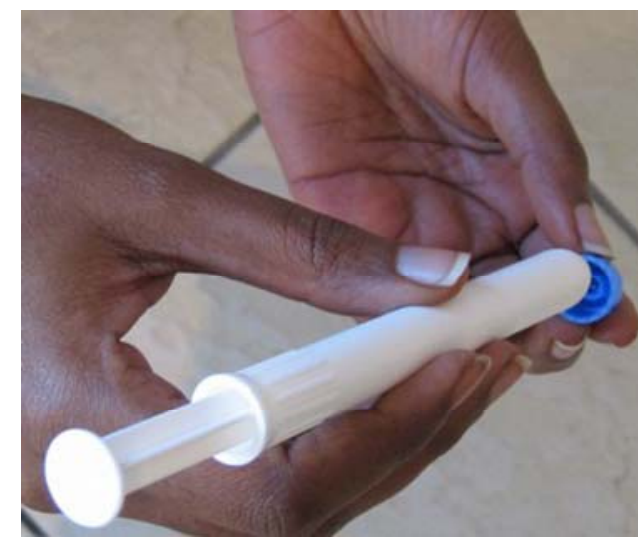

Figure 3. Traditional vaginal applicator.

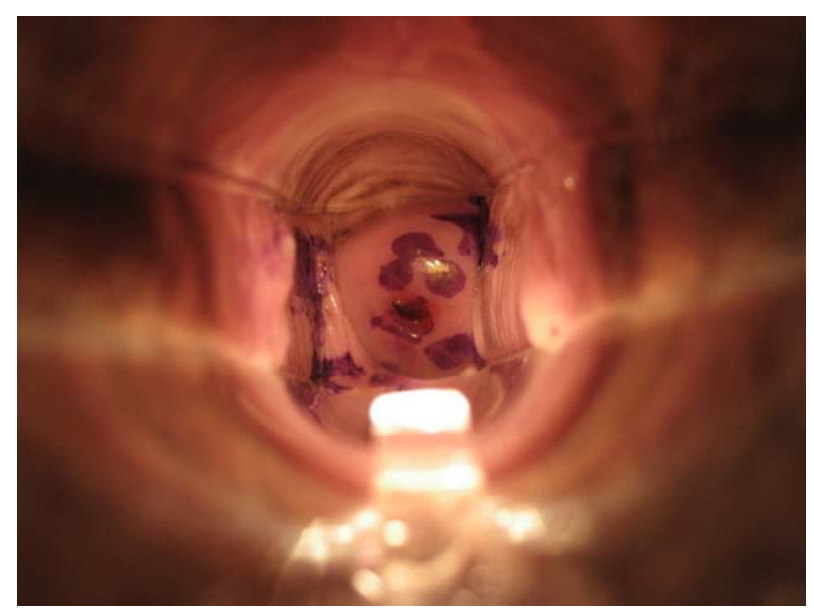

Figure 4. Retained gel over the cervix and vagina.

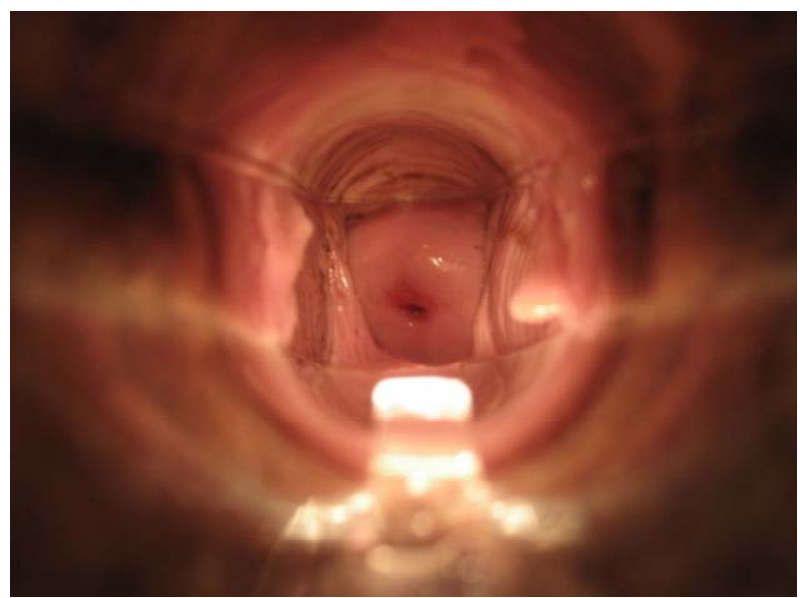

Figure 5. The vagina. 
Table 1. Comparison between distribution and retention of the vaginal lubricant.

\begin{tabular}{ccc}
\hline $\begin{array}{c}\text { 24 hours } \\
\text { after gel application }\end{array}$ & $\begin{array}{c}\text { The FemCap } \\
\text { Cervix }\end{array}$ & $\begin{array}{c}\text { The Vaginal Applicator } \\
\text { Stained Vaginal Lubricant applied with the FemCap once } \\
\text { before intercourse }\end{array}$ \\
$\begin{array}{c}\text { Spper vagina } \\
\text { Introitus }\end{array}$ & $\begin{array}{c}\text { Stained lubricant applied with the vaginal applicator twice } \\
\text { before and after intercourse }\end{array}$ \\
Present with many bare spots (Figure 4) & Absent (Figure 5) \\
\hline
\end{tabular}

\section{Discussion}

According to the CAPRISA 004 study [1] report, "Inadequate adherence is the most serious challenge to obtaining an accurate estimate of product efficacy. "This highlights the need to seek higher levels of adherence and effectiveness with Tenofovir gel and to develop other effective prevention strategies for use in combination with Tenofovir gel”.

Based on the CAPRISA 004 [1] result report above, we investigated the feasibility and acceptability of an alternative to the vaginal applicator (Figure 3) used in CAPRISA 004. We agree with the authors of CAPRISA 004 study that poor adherence with Tenofovir application was a major factor in its partial success. Prevention of the spread of HIV transmission should be the front and centre of research studies. Adherence with Tenofovir gel application significantly affected HIV seroconversion in the CAPRISA 004 study.

This study validate and verify our previous pilot study [11] to improve on the adherence and retention and thus the effectiveness of Tenofovir gel microbicide application. We utilized the FemCap delivery system to deliver a stained vaginal lubricant as a substitute for Tenofovir gel. We realize that the number of participants is small to make a judgment about levels of compliance in relation to type of product, however a larger study is planned that will provide the tissue concentration level of Tenofovir and a meaningful statistical analysis.

This methodology allowed us to compare compliance, as well as the presence or absence of the stained vaginal lubricant over the cervix and vagina after 24 hours. Future studies will be conducted to show the exact inhibitory level of Tenofovir in the epithelial surface of the cervix and vagina at 6,12 , and 24 hours when applied with the applicator and FemCap.

Participants were counseled on the importance of honest and truthful reporting for each and every time they used the vaginal applicator and the FemCap. The majority of women, $80 \%$, liked the fact that they only needed to use the FemCap once before intercourse and reapply it after intercourse only if they neglected to use it before intercourse. They also liked the lack of leakage of the stained lubricant from the vagina and the FemCap is proven to prevent pregnancy and thus prevent mother -to child HIV transmission and could maintain contact between the microbicide and the cervix and vagina for up to 24 hours.

Even a modest enhancement of adherence and retention of the gel, due to the higher acceptability of the FemCap/ microbicide delivery system, has the potential to increase Tenofovir effectiveness and save millions of lives. The FemCap is well accepted among American women [20] as well as African women [11]. If this simple strategy could reduce the risk of STI/HIV transmission, it would have an enormous impact worldwide. Another advantage of using the FemCap is the prevention of unintended pregnancy and thus prevents the transmission of the HIV virus from mother-to-child. Future studies are needed to proof this concept and explore the safety of Tenofovir gel application using this novel device.

\section{Conclusions}

This pilot study illustrated, that, participants had higher acceptability of the FemCap due to elimination of leakage and the single application of the vaginal lubricant, versus two applications with the vaginal applicator. The use of the FemCap, can prevent pregnancy, HIV motherto-child transmission, and will enhance compliance, distribution and retention of gel over the cervix that may potentially prevent STIs and increase the efficacy of Tenofovir gel.

\section{Acknowledgements}

This paper was prepared at the request of Jing Guo the Editor of World Journal of AIDS (WJA). We are indebted to the women who consented to participate in this research study, and to Dr. Ramez Eskander who edited this manuscript. No funding was requested or received from any institution. The authors are responsible for the content of this article

\section{REFERENCES}

[1] Q. A. Karim, S. S. A. Karim, J. A. Frohlich, et al., "Effectiveness and Safety of Tenofovir Gel, an Antiretroviral Microbicide, for the Prevention of HIV Infection in Women,” Science, Vol. 329, No. 5996, 2010, pp. 1168- 
1174.

[2] T. Silvio, D. Martinelli, C. Germinario, et al., "Determining Factors for Condom Use: A Survey of Young Italian Adults," European Journal of Contraception Reproductive Health Care, Vo. 15, No. 1, 2010, pp. 24-30. doi:10.3109/13625180903427683

[3] R. A. Crosby, W. L. Yarber, C. A. Graham, et al., "Does It Fit Okay? Problems with Condom Use as a Function of Self-Reported Poor Fit," Sexually Transmitted Infections, Vo. 86, No. 1, 2010, pp. 36-38. doi:10.1136/sti.2009.036665

[4] World Savvy Monitor, "HIV/AIDS and Sexually Transmitted Infections,” Global Status of Women, 2009. http://worldsavvy.org/monitor/index.php?option=com_co ntent\&view=article\&id=582\&Itemid=1029

[5] J. Pudney, A. J. Quayle and D. J. Anderson, "Immunological Microenvironments in the Human Vagina and Cervix: Mediators of Cellular Immunity Are Concentrated in the Cervical Transformation Zone,” Biology of Reproduction, Vol. 73, No. 6, 2005, pp. 1253-1263. doi:10.1095/biolreprod.105.043133

[6] M. Laga, A. Manoka, M. Kivuvu, et al., "Non-Ulcerative Sexually Transmitted Diseases as Risk Factors for HIV-1 Transmission in Women: Results from a Cohort Study," AIDS, Vol. 7, No. 1, 1993, pp. 95-102. doi:10.1097/00002030-199301000-00015

[7] L. Van Damme, G. Ramjee, M. Alary, et al., "Effectiveness of COL-1492, a Nonoxynol-9 Vaginal Gel, on HIV-1 Transmission in Female Sex Workers: A Randomised Controlled Trial," Lancet, Vol. 360, No. 9938, 2002, pp. 971-977. doi:10.1016/S0140-6736(02)11079-8

[8] L. Van Damme, R. Govinden, F. Mirembe, et al., "Lack of Effectiveness of Cellulose Sulfate Gel for the Prevention of Vaginal HIV Transmission," The New England Journal of Medicine, Vol. 359, 2008, pp. 463-472. doi:10.1056/NEJMoa0707957

[9] S. Salim, "HIV Prevention Gel Proven Ineffective in Largest Clinical Trials," Centre for the AIDS Program of Research in South Africa (CAPRISA), University of KwaZulu-Natal, 2009, pp. 527-529.

[10] S. Skoler-Karpoff, G. Ramjee, K. Ahmed, et al., "Effi- cacy of Carraguard for Prevention of HIV in Women in South Africa," Lancet, Vol. 372, No. 9654, 2008, pp. 1977-1987. doi:10.1016/S0140-6736(08)61842-5

[11] A. Shihata and S. Brody, "HIV Prevention by Enhancing Compliance of Tenofovir Microbicide. Using a Novel Delivery System," HIV \&AIDS Review, Vol. 9, No. 4, 2010, pp. 107-110.

[12] H. Carcio, M. Clarke Secor and R. Koeniger-Donohue, "Advanced Health Assessment of Women: Clinical Skills and Procedures, Chapter 15 The FemCap,” Springer Publishing Company, New York, 2010, pp. 271-278.

[13] R. Koeniger-Donohue, “The FemCap: A Non-Hormonal Contraceptive," Women's Health Care, Vol. 5, No. 4, 2006, pp. 79-91.

[14] A. Shihata, "The FemCap ${ }^{\mathrm{TM}}$, a New Contraceptive Choice,” European Journal of Contraception Reproductive Health Care, Vol. 3, 1998, pp. 160-166.

[15] D. Shoupe and S. Kjos, "The Handbook of Contraception, Barrier Contraceptives,” Humana Press, Totowa, 2006, pp. 147-177.

[16] A. Shihata, "Microbicides Delivery System," 5th European Congress on Tropical Medicine and International Health, Amsterdam, 24-28 May 2007, pp. 131-134.

[17] A. Shihata, "Novel Delivery System for Microbicides," 2007 National HIV Prevention Conference, Atlanta, 2-5 December 2007, p. 378.

[18] A. Shihata, "New FDA Approved Woman-Controlled, Latex-Free Barrier Contraceptive Device 'FemCap ${ }^{\mathrm{TM}}$,', Fertility and Sterility, Vol. 5, No. 103, 2004, pp. 303-330.

[19] A. Shihata and E. Gollub, "Acceptability of a New Intravaginal Barrier Contraceptive Device FemCap,” Contraception, Vol. 46, No. 6, 1992, pp. 511-519. doi:10.1016/0010-7824(92)90115-A

[20] A. van der Straten, N. Sahin-Hodoglugil, K. Clouse, S. Mtetwa and M. Z. Chirenje, "Feasibility and Potential Acceptability of Three Cervical Barriers among Vulnerable Young Women in Zimbabwe," Journal of Family Planning and Reproductive Health Care, Vol. 36, No. 1, 2010, pp. 13-19. 